\title{
Spatially controlled doping of silver in chalcogenide glass by thermal, photo and electron beam effects
}

\author{
Katrina A. Morgan, Christopher Craig, Andrea Ravagli, Ed Weatherby, Armen Aghajani, Daniel W. \\ Hewak \\ Optoelectronics Research Centre, University of Southampton, Highfield Campus, Southampton, UK, SO17 1BJ
}

For the first time, silver doping of bulk Gallium-Lanthanum-Sulphide (GLS) and Gallium-Lanthanum-SulphurSelenide (GLSSe) glass is demonstrated, presenting potential for a huge array of optoelectronic applications. Previously, silver doping of chalcogenide glasses were mostly based upon toxic, arsenic containing thin films [13]. GLS and GLSSe, however, offer a toxic free alternative, made by a safer and more economic technique, with infrared and nonlinear optic capacity [4].

GLS and GLSSe bulk glass were manufactured using a melt-quench technique with high purity precursors. A silver layer of $10 \mathrm{~nm}$ was deposited onto $1 \mathrm{~mm}$ thick polished GLS and GLSSe glass using electron-beam evaporation. Ag/GLS and Ag/GLSSe samples then underwent three different treatments: heated at $300^{\circ} \mathrm{C}$ with no UV for 24 hours (1), heated at $100^{\circ} \mathrm{C}$ with UV exposure (55 W, $312 \mathrm{~nm}$ ) for 24 hours (2), and no UV or heat exposure for 50 days (3).

FTIR, UV/VIS, SEM and Raman spectroscopy were performed on all samples to investigate the effects of thermal and photo-doping. Reference samples of GLS and GLSSe without silver were used for baselines. The transmission spectra for all samples are shown in Fig. 1 (a) and (b). From this it can be seen that thermal-doping (1) is much more efficient than photo-doping (2) as evidenced by a higher transmission, similar to that of the reference blank glass, for both GLS and GLSSe. The low transmission seen for samples after the UV exposure (2) and for samples without any heat or UV exposure (3) is attributed to high reflection caused by silver remaining on the surface of the glass, which is confirmed by SEM and Raman. When taking into consideration UV exposure (2), the higher transmission of GLS compared to GLSSe indicates that Ag more readily migrates into GLS than GLSSe due to the difference in composition [5]. We also note that in the absence of UV and heat exposure (3), a small amount of doping has occurred over time as seen visually.

Previous studies have successfully manipulated the migration of silver in an array of glasses via electron beam irradiation. It is thought the silver is induced by the electric field generated by electrons, thus enabling high resolution features to be created [3]. In order to investigate this, GLS and GLSSe after heat exposure (1) were irradiated using a $20 \mathrm{kV}$ SEM beam. Initially the SEM images of the surface appear smooth, in agreement with absence of silver peaks in the Raman spectra, indicating the full migration of the entire silver layer into the glass. When the SEM beam is focused, however, for a prolonged period of time, photo-migration and photo-deposition occur, as shown in Fig. 1 (c) and (d) whereby silver emerges out of the glass onto the surface, in the beam area.
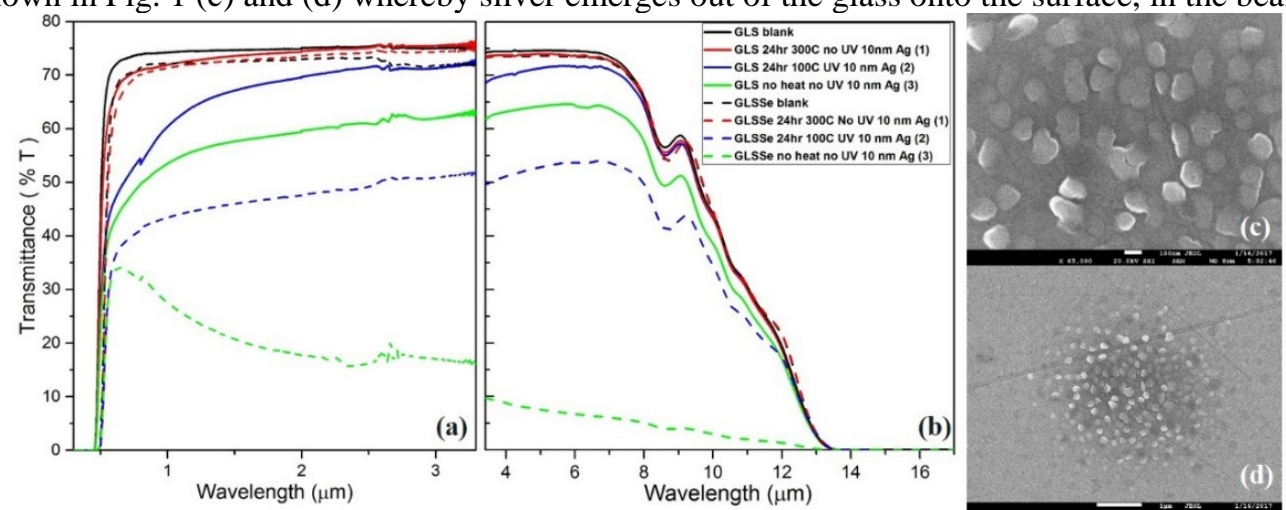

Fig. 1 Transmission spectra of GLS and GLSSe with photo and thermal treatments, measured by (a) UV/VIS and (b)

FTIR. SEM images of thermally treated GLSSe with silver emerging after 1 min focus at (c) x65k (d) x17k mag.

In this work, successful thermal-doping, photo-migration and re-deposition of silver in bulk GLS and GLSSe glass has been demonstrated, opening up a huge array of potential applications including high resolution lithography. Further investigations will be conducted into thin film silver doped GLS and GLSSe, on flexible substrates, applicable for roll-to-roll (R2R) fabrication thus making it ideal for wearable and flexible technology.

\section{References}

[1] J. Tasseva, R. Todorov, T. Babeva, and K. Petkov, "Structural and optical characterization of Ag photo-doped thin As40S60-xSex films for non-linear applications," J. Opt. 12,065601 (2010).

[2] A. V. Kolobov, Photo-Induced Metastability in Amorphous Semiconductors, Ch. 11, (John Wiley \& Sons, 2006).

[3] N. Yoshida, and K. Tanaka, "Ag migration in Ag-As-S glasses induced by electron-beam irradiation," J. Non-Cryst. Solids. 210, 119-129 (1997).

[4] A. Ravagli, C. Craig, D. W. Hewak, "Ga-La-S glass for UV and IR applications," IONS, Quebec, CA (2016).

[5] M. Frumar, T. Wagner, "Ag doped chalcogenide glasses and their applications," Curr. Opin. Solid State Mater. Sci. 7, 117-126 (2003). 